\title{
Recommendations for epidemiologic studies of aging populations in a changing climate
}

\author{
Sindana D. Ilango ${ }^{1,2} \cdot$ Sara McElroy ${ }^{1,2}$ (1) $\cdot$ Lara Schwarz $^{1,2}$ \\ Received: 29 April 2019/Revised: 16 July 2020 / Accepted: 12 August 2020 / Published online: 30 August 2020 \\ (C) Swiss School of Public Health (SSPH+) 2020
}

The health effects of climate change on an aging population are not well understood. Maximizing healthy aging to improve quality of life at older ages is a pressing public health priority as the proportion of adults over 65 increases, from $9 \%$ in 2020 to an expected $16 \%$ in 2050 (United Nations Department of Economic and Social Affairs 2019). Ambient environmental exposures and extreme weather events pose health risks that can be addressed by population-level interventions (World Health Organization (WHO) 2017). In the context of climate change, extreme weather conditions and events are becoming more frequent, more severe, and less predictable; all are linked to adverse health outcomes like heart disease, respiratory conditions, and premature mortality (Åström et al. 2011; Gamble et al. 2013). Older adults are less resilient to climate change because they are more likely than younger populations to have impaired physical function, comorbidities, compromised immune systems, and to be more socially isolated (Gamble et al. 2013). This is why we have chosen to underline the need to study the effects of climate change on older adults. Specifically, we encourage conducting epidemiologic studies on the impact of climate change in lowand middle-income countries (LMIC) and targeting research questions to inform public health decisions.

This Editorial is part of the series "Young Researcher Editorial", a training project of the Swiss School of Public Health.

The Editorial is published to mark the 15th anniversary of the Swiss School of Public Health.

Sindana D. Ilango silango@ucsd.edu

1 Family Medicine and Public Health, University of California, San Diego, La Jolla, CA, USA

2 School of Public Health, San Diego State University, San Diego, CA, USA
Previous epidemiologic studies on climate change and aging-related health were predominantly conducted in high-income regions with temperate climates and accessible weather and health data (Ostro et al. 2018). These have evaluated and informed policies aiming to decrease the health burden imposed by climate change. For example, in 2009-2010, New York City prevented 8 heat-related illnesses per 10 days in a population of Medicare beneficiaries over 65 years old by changing its heat emergency plan in 2008 to meet thresholds suggested by local epidemiologic studies (Benmarhnia et al. 2019). But findings from research in high-income regions may not be generalizable to the aging populations in LMIC. Research like this is less common in LMIC, where the fertility patterns, increased migration, and longer life expectancy are also increasing the proportion of older adults (United Nations Department of Economic and Social Affairs 2019); the population of the elderly is expected to rise from 8 to $14 \%$ by 2050 (United Nations Department of Economic and Social Affairs 2019). Although LMIC are the smallest per capita contributors of greenhouse gases, they are heavily burdened by the effects of climate change. Because many LMIC lack robust infrastructure to mitigate the effects of extreme weather conditions and events, they are highly vulnerable to climate change and the effect of these changes is exacerbated by health and socioeconomic disparities (Eckstein et al. 2019). To address these problems and maximize benefits in target LMIC populations, we need to develop location- and population-specific interventions, e.g., identifying local thresholds for early warning systems.

In LMIC, limited data makes studying climate-related health effects among older adults challenging. Many LMIC do not have many weather monitoring stations available to collect data for analysis. To overcome these limitations, we recommend deploying emerging technologies (e.g., satellite data) to estimate exposures in under-monitored areas (Pinder et al. 2019). Researchers can use global remote 
sensing data from satellites and publicly available platforms to monitor environmental exposures and examine exposures at a fine scale (Ostro et al. 2018). They could then overcome the limitations of sparse ground-station data and limited routine health records, and link these exposure estimates to health surveys (e.g., Demographic Health Surveys) to study the effects of environmental exposures on health among older adults (Green et al. 2018).

By carefully targeting research questions using thoughtful exposure classifications, absolute measures of association, and by identifying disproportionately vulnerable subgroups, researchers can more readily translate public health research to policy. Exposure measurements should be categorized using region-specific thresholds to inform when advisory warning system are activated. For example, heat warning systems can be triggered when local temperatures exceed a threshold estimated to harm the health of older people. Because environmental conditions and extreme weather events are potentially modifiable risk factors, we encourage researchers to design studies that measure associations on an absolute scale with additive models or convert relative measures to their absolute equivalents (Knol et al. 2011). Evidence suggests absolute measures (e.g., risk difference) are more appropriate than relative measures (e.g., risk ratio) for defining criteria for activating early warning systems for heat waves (McElroy et al. 2020). In order to gain the greatest population health benefit, researchers should prioritize studies that will help them estimate the number of cases attributable to a specific event. Finally, analyses to identify vulnerable subpopulations (e.g., stratification) and assess spatial heterogeneity are crucial to guide public health agencies on delegating scarce resources.

Expanding public health research on climate change and aging-related health outcomes can directly benefit older adults and indirectly the whole population. We recommend a greater emphasis on studies in LMIC and targeted epidemiologic research studies with findings that can be applied in policy settings. In combination, these approaches will help identify health disparities and inform public health policy designed to improve population health in a changing climate.

\section{References}

Åström DO, Bertil F, Joacim R (2011) Heat wave impact on morbidity and mortality in the elderly population: a review of recent studies. Maturitas 69(2):99-105

Benmarhnia T, Schwarz L, Nori-Sarma A, Bell ML (2019) Quantifying the impact of changing the threshold of New York City heat emergency plan in reducing heat-related illnesses. Environ Res Lett. https://doi.org/10.1088/1748-9326/ab402e

Eckstein D, Künzel V, Schäfer L, Winges M (2019) Global climate risk index 2020. Germanwatch. Available at: https://german watch.org/sites/germanwatch.org/files/20-2-01e\%20Global\%20 Climate\%20Risk\%20Index\%202020_14.pdf

Gamble JL, Hurley BJ, Schultz PA, Jaglom WS, Krishnan N, Harris M (2013) Climate change and older Americans: state of the science. Environ Health Perspect 121(1):15-22. https://doi.org/ 10.1289/ehp. 1205223

Green H, Bailey J, Schwarz L, Vanos J, Ebi K, Benmarhnia T (2018) Impact of heat on mortality and morbidity in low and middle income countries: a review of the epidemiological evidence and considerations for future research. Environ Res 2019(171):80-91. https://doi.org/10.1016/j.envres.2019.01.010

Knol MJ, VanderWeele TJ, Groenwold RHH, Klungel OH, Rovers MM, Grobbee DE (2011) Estimating measures of interaction on an additive scale for preventive exposures. Eur J Epidemiol 26(6):433-438. https://doi.org/10.1007/s10654-011-9554-9

McElroy S, Schwarz L, Green H et al (2020) Defining heat waves and extreme heat events using sub-regional meteorological data to maximize benefits of early warning systems to population health. Sci Total Environ 721:137678. https://doi.org/10.1016/j.scito tenv.2020.137678

Ostro B, Spadaro JV, Gumy S et al (2018) Assessing the recent estimates of the global burden of disease for ambient air pollution: methodological changes and implications for low-and middle-income countries. Environ Res 166:713-725

Pinder RW, Klopp JM, Kleiman G, Hagler GSW, Awe Y, Terry S (2019) Opportunities and challenges for filling the air quality data gap in low- and middle-income countries. Atmos Environ 215(February):116794. https://doi.org/10.1016/j.atmosenv.2019. 06.032

United Nations Department of Economic and Social Affairs (2019) Revision of world population prospects

World Health Organization (WHO) (2017) Global strategy and action plan on ageing and health. https://doi.org/10.1136/hrt.2006. 103895

Publisher's Note Springer Nature remains neutral with regard to jurisdictional claims in published maps and institutional affiliations. 